\title{
ESTRATEGIA DE GESTIÓN PARTICIPATIVA TERRITORIAL PARA FORTALECER EL TURISMO CULTURAL: ZONA PATRIMONIAL DEL MUNICIPIO DE LEÓN, NICARAGUA
}

\section{TERRITORIAL PARTICIPATORY MANAGEMENT STRATEGY TO STRENGTHEN CULTURAL TOURISM: HERITAGE AREA OF THE MUNICIPALITY OF LEÓN, NICARAGUA}

\author{
Vianney del Carmen Fornos García', Meylin Alvarado Sánchez ${ }^{2}$ \\ 1 Universidad Nacional Autónoma de Nicaragua (UNAN), León, Nicaragua \\ 2 Universidad Nacional de Costa Rica (UNA), Heredia, Costa Rica
}

Correspondência para: Meylin Alvarado Sánchez (malvara@una.cr)

doi: 10.12957/geouerj.2019.45773

Recebido em: 4 out. 2019 | Aceito em: 1 nov. 2019

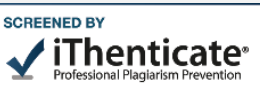

\section{RESUMEN}

El Consejo Internacional de Monumentos y Sitios Históricos (ICOMOS) de la Organización de las Naciones Unidas para la Educación, la Ciencia y la Cultura (UNESCO), por medio de la Carta Internacional Para la Conservación de Poblaciones y Áreas Urbanas Históricas (Carta de Washington, 1987) indica que todos los conjuntos urbanos del mundo, son la expresión material de la diversidad de las sociedades a lo largo de la historia. Por lo que es necesario definir objetivos, métodos e instrumentos para conservar la calidad de las poblaciones de estas y favorecer la armonía entre la vida individual y colectiva en las mismas, perpetuando el patrimonio de la humanidad. Al respecto, la ciudad de León de Nicaragua, alberga el patrimonio histórico-cultural más grande y diversos del país y de gran importancia en Centroamérica, por lo que el objetivo principal de esta publicación es mostrar algunos resultados, de la gestión participada para el aprovechamiento sostenible los recursos culturales en la zona patrimonial de esta ciudad; donde mediante procesos de coordinación entre el sector público, privado y la población local, se definieron diferentes líneas estratégicas para el turismo cultural sostenible y su zonificación, como parte de la propuesta del plan de ordenamiento territorial del centro Histórico de León, para el año 2017.

Palabras-clave: Patrimonio Cultural. Gestión participada. Turismo Cultural. Zona Patrimonial.

\begin{abstract}
The International Council of Monuments and Historic Sites (ICOMOS) of the United Nations Educational, Scientific and Cultural Organization (UNESCO), through the International Charter for the Conservation of Populations and Historical Urban Areas (Letter of Washington, 1987) indicates that all the urban ensembles of the world are the material expression of the diversity of societies throughout history. Therefore, it is necessary to define objectives, methods and instruments to preserve the quality of their populations and to promote harmony between individual and collective life in them, perpetuating the heritage of humanity. The city of León Nicaragua houses the largest and most diverse historical and cultural heritage of Nicaragua and the most important in Central America. The main objective of this article is to show the partial results of the participatory management for the sustainable use of cultural resources in the heritage area of this city. By implementing coordination processes between public, private and local groups, different strategic lines were defined for the implementation of sustainable cultural tourism and zoning the city's cultural heritage area as part of a proposal for the territorial planning plan of the Historic Center of León for the year 2017.
\end{abstract}

Keywords: Cultural Heritage. Participative Management. Cultural Tourism. Heritage Zone.

\section{INTRODUCCIÓN}


Nicaragua, como muchos de los países de la región latinoamericana, ha visualizado el turismo como de sus principales actividades económicas, participando de las potencialidades turísticas de Centroamérica, según, López (2005) los principales productos de esta región, se basan en los recursos culturales (ligado a la civilización precolombina y a la colonización europea en esta región) y en la naturaleza (playas, áreas protegidas, lagos y volcanes), presentando diversos destinos turísticos, como las ciudades coloniales. Entre estas destaca la nueva ciudad de León de Nicaragua, fundada en el año 1610, en plena época de la colonia española en la región centroamericanas, pertenece al municipio de León, ubicado al noroccidente del país, a $92 \mathrm{~km}$ de la capital Managua, con una Extensión Territorial: 820. 19 km cuadrados y según el Instituto Nacional de Estadísticas y Censos (INEC), en su el Censo poblacional y de vivienda (2005), esta cuenta con 208,000 habitantes, predominantemente urbana, La Figura 1, muestra la ubicación de León en Nicaragua.

Esta es una ciudad que alberga en sí un cuantioso patrimonio arquitectónico, históricos, cultural, natural y simbólico de gran valor para el país y la región, por tanto se representa en ella un enclave de vital importancia para la realización de muchas investigaciones en la temática y proyectos de conservación y recuperación de la herencia colonial, incluyendo en estos los elementos socio-culturales que se entretejen en la dinámica activa de la ciudad. De tal manera que cuenta con dos Sitios Patrimonios de la Humanidad, declarados por la UNESCO, como son: las Ruinas de León Viejo, uno de los más antiguos asentamientos coloniales españoles en el continente americano y la Real Basílica Catedral de la Asunción, conocida como la Catedral de León, que fue construida entre finales del siglo XVIII y principios del siglo XIX, con un estilo considerado ecléctico. La Figura 2, muestra una imagen de la Catedral de León.

A partir de una gestión turística compartida, entre el sector público, el sector privado, la cooperación internacional y la participación activa de la población local (Apoyo Técnico de la Asociación Medina ${ }^{1}$, el Municipio de León, la UNAN León, los actores locales y con financiamiento de la Unión Europea )se planteó el proyecto denominado "Propuesta Estratégica para el Aprovechamiento Sostenible de los Recursos Culturales de la Zona Patrimonial de la Ciudad de León, Nicaragua” Aquí se presentan los resultados de esa estrategia de gestión participativa, donde se definieron diferentes líneas estratégicas

\footnotetext{
${ }^{1}$ Asociación Medina: Empresa consultora nicaragüense, encargada de coordinar el proyecto.
} 
participativas y la zonificación correspondiente, como parte de la propuesta del Plan de Ordenamiento

Territorial del Centro Histórico de la Ciudad de León, Nicaragua, para el año 2017.

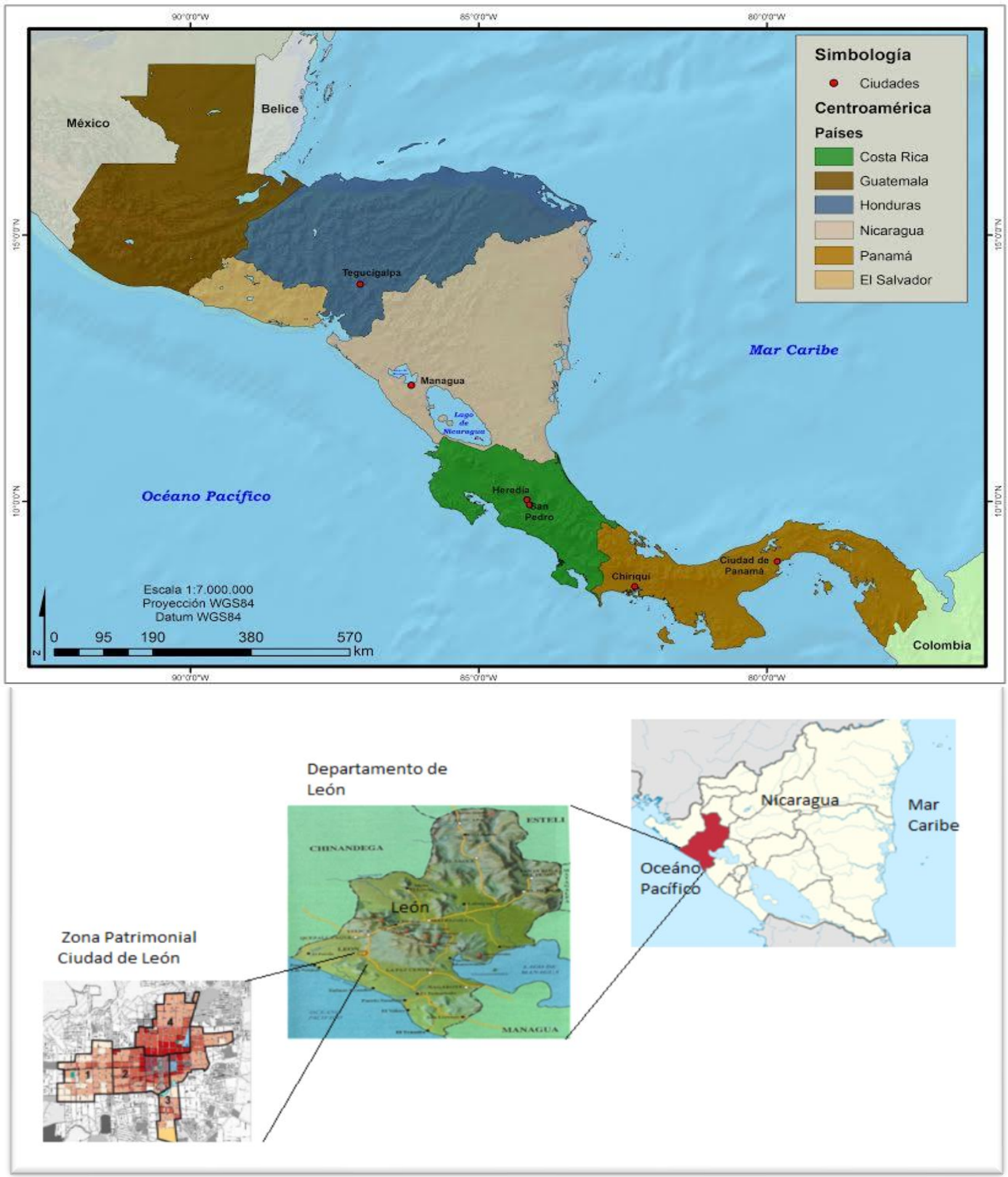

Figura 1. Ubicación de la Ciudad de León, en Nicaragua, Centroamérica. Fuente: Elaboración propia. Edición Cartográfica mapa de Centroamérica, Lic. Karla Mora Aparicio, 2016. 


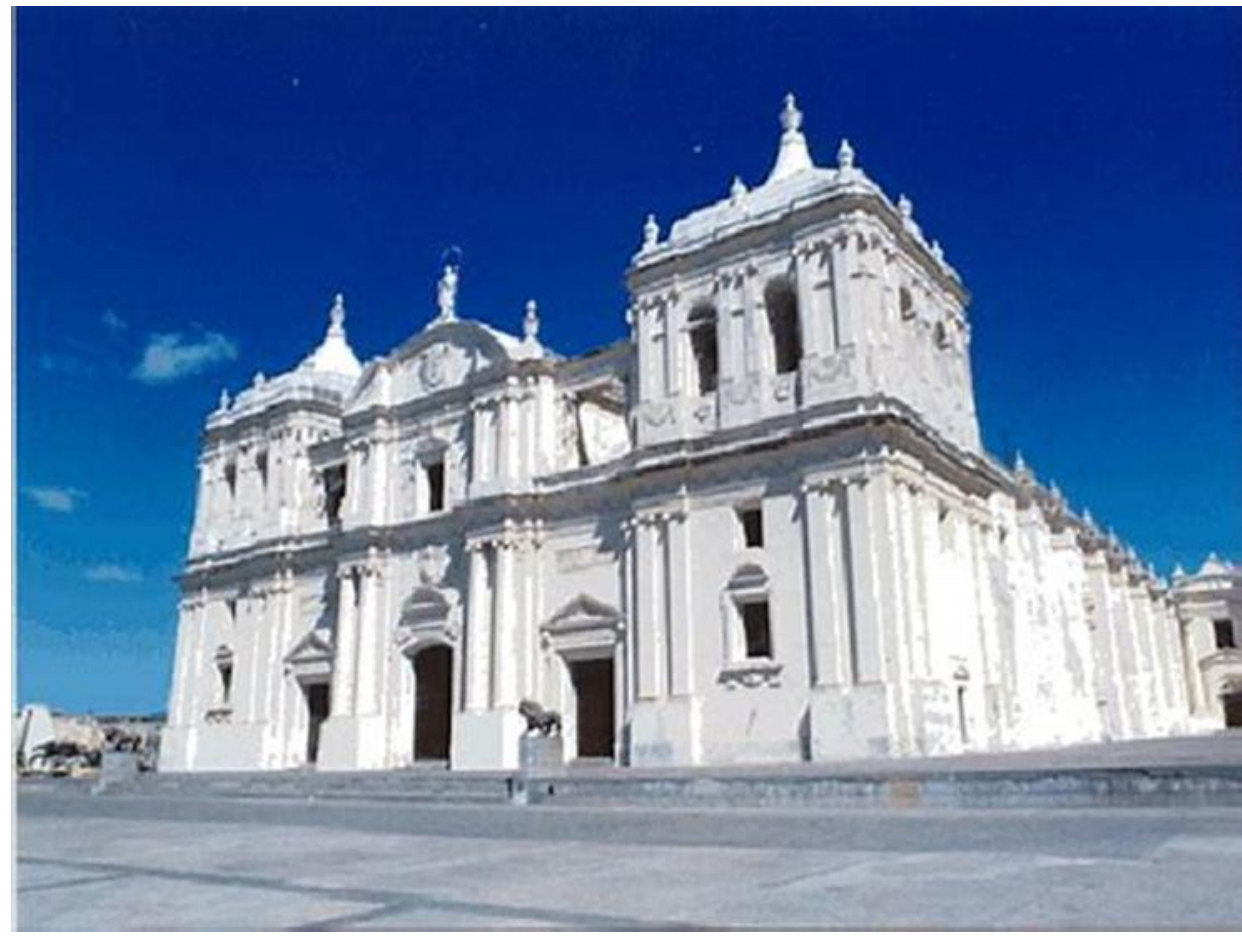

Figura 2. la Catedral de León, Sitio Patrimonial de la Humanidad, declaradas por la UNESCO, construida entre finales del siglo XVIII. Fuente: Proyecto Estrategia de Gestión Sostenible y Participativa del Centro Histórico de León, 2017.

\section{Patrimonio Territorial y Turismo Cultural.}

Desde el punto de vista conceptual, la palabra patrimonio, deriva del término en latín "patrimonium" y su significado tiene relación con la herencia y además posee un componente temporal, Según González y Castañeda (2011), esta obliga a la retrospección. a mirar al pasado, a meditar sobre lo que se dejará a los hijos. La concepción de esta herencia, frecuentemente vinculada con lo material, debe explorarse y repensarse en términos más subjetivos e intangibles, porque se hereda también la parte menos visible de la cultura: como conductas, hábitos, costumbres, valores, identidades, historia, saberes, modos de vida de un territorio. Por su parte De Carli, (2007) lo define como el conjunto de bienes culturales y naturales, tangibles e intangibles, generados localmente, y que una generación hereda y transmite a la siguiente, con el fin de preservarla y acrecentarla.

Según ILAM (2017), La UNESCO, como ente rector a nivel mundial, en la declaratoria de Patrimonio de la Humanidad o Patrimonio Mundial en 1972, presenta una subclasificación del concepto de patrimonio, donde distingue tres tipos de patrimonio: el patrimonio Cultural, subdividido en Tangible (mueble e inmueble) e Intangible, como pinturas, libros, objetos domésticos, monumentos, sitios 
históricos y arquitectónicos, saberes, formas de expresión y lugares. Seguido por el patrimonio Natural Cultural, que incluye vestigios arqueológicos o históricos, fósiles paleontológicos, subacuáticos y paisaje cultural. La tercera clasificación es la del patrimonio Natural, constituido por formaciones físicas y biológicas, formaciones geológicas y fisiográficas que sean hábitats de especies amenazadas y zonas naturales estrictamente delimitadas de valor universal excepcional.

Respecto al Patrimonio Cultural, en la Conferencia Mundial de la UNESCO, sobre el Patrimonio Cultural, celebrada en México en el año 1982, se dio la siguiente definición:

"El Patrimonio Cultural de un pueblo comprende las obras de sus artistas, arquitectos, músicos, escritores y sabios, así como las creaciones anónimas, surgidas del alma popular, y el conjunto de valores que dan sentido a la vida, es decir, las obras materiales y no materiales que expresan la creatividad de ese pueblo; la lengua, los ritos, las creencias, los lugares y monumentos históricos, la literatura, las obras de arte y los archivos y bibliotecas.

Esta conceptualización de Patrimonio Cultural, remite a territorios específicos y con criterios muy delimitados; haciendo alusión al concepto al enfoque territorial, permite dilucidar recursos naturales de gran valor cultural para las comunidades rurales y sus visitantes, extender esta visión conlleva a incluir aquellos espacios naturales comunitarios como ríos, bosques, montañas y otros de valor simbólico que forman parte de su patrimonio territorial, con potencial turístico. Situación que lo convierte en un bien merecedor de una adecuada conservación, valorándolo para el conocimiento de un territorio, de una época y de un estilo de vida, resguardando las memorias y las experiencias humanas significativas, lo que justifica las metodologías participativas, que se han implementado en este proyecto para la ciudad de León.

Para este tipo de gestión del patrimonio como atractivo turístico, también se requiere de un proyecto de “desarrollo endógeno”, donde el producto turístico se base en función de las fortalezas y necesidades locales, no en las exigencias de la demanda turística; tomando en cuenta también su fragilidad y la necesidad de un control y gestión local de los recursos. Basados en el paradigma de la sostenibilidad. De acuerdo con el Manual de la UNESCO: Gestión del Patrimonio Mundial Cultural, 2013, para una adecuada gestión del Patrimonio cultural, se requiere de un plan de sostenibilidad, el cual debe basarse en dos enfoques: 
- El primero basado en el interés por sostener el Patrimonio, considerándolo como un fin en sí y como parte de los recursos ambientales/culturales que deben protegerse y trasmitirse a las generaciones futuras para garantizar su desarrollo (intrínseco).

- El segundo, entendiéndolo como la contribución que el patrimonio y su conservación pueden aportar a las dimensiones ambientales, sociales y económicas del desarrollo sostenible (instrumental).

La sostenibilidad turística, en la gestión patrimonial de la ciudad de León, fue abordado desde ambos enfoques, porque desde el primero, los beneficios socioeconómicos derivados de los bienes del patrimonio serían desde luego deseables, pero no estrictamente necesarios para justificar su conservación y los resultados de adoptar el segundo enfoque, donde el patrimonio contribuya a la sostenibilidad, son importantes para el sector, porque suponen un cambio del planteamiento extremadamente filosófico y ético de la conservación, donde se considera que hay que conservar porque "así debe ser". Las decisiones sobre la conservación del Patrimonio ya no se confiarían solamente a los expertos en la disciplina, sino que se debatirían entre muchas partes implicadas, que concertarían compromisos basados en argumentos sólidos y objetivos compartidos, de equipos multidisciplinarios.

Esto permite reafirmar el valor cultural del Patrimonio haciendo más explícita su contribución al bienestar, la felicidad de la sociedad, y estudiar las condiciones que permitirían que desde la puesta en valor se aporte a la sostenibilidad ambiental, social y económica, a través del uso turístico como una alternativa incluyéndose, como un producto de turismo cultural. En ICOMOS, adoptada en Bruselas en 1976), se define este tipo de turismo como, aquella actividad que tiene por objeto, entre otros fines, el conocimiento de monumentos y sitios histórico-artísticos. Por su parte la Secretaría de Turismo de México (2002:4) lo define como:

El turismo cultural se comporta como un elemento dinamizador del patrimonio y las comunidades, genera reconocimiento y creación de sentimiento de orgullo comunitario, y es factor de divulgación del patrimonio. Como valor para la cultura, genera recursos para la conservación y beneficia a las comunidades receptoras, motiva a las comunidades en la gestión de su patrimonio y crea conciencia del valor de los diferentes "patrimonios locales" entre los turistas. 
Este tipo de turismo, justifica los esfuerzos dirigidos hacia una adecuada gestión del patrimonio cultural, para su mantenimiento, protección y puesta en valor turístico, con el fin de obtener beneficios socioculturales y económicos, para toda la población involucrada.

\section{Abordaje Metodológico}

La metodología implementadas en esta investigación, fue de tipo participativa, rescatando prioritariamente los valores, considerando los principales actores de la ciudad de León, en torno a la interacción ciudadana, sus costumbres, tradiciones, vida cotidiana y desarrollo socio-económico, enfocado en promover varios sectores, sobre todo el sector del patrimonio, dada la percepción de este como bien compartido por un territorio.

Entendiendo por metodologías participativa, aquellas que conllevan al protagonismo de las comunidades, mediante procesos participativos. Arnanz $(2012,4)$ donde:

\footnotetext{
A través de un proceso participativo, que la comunidad y sus diferentes sectores sociales con intereses legítimos en un proyecto, programa o política de desarrollo, intervienen en ellos y van adquiriendo un protagonismo cada vez mayor en el análisis de su propia realidad, en la toma de decisiones y en la gestión de los recursos. De esta manera se convierten en actores determinantes de su propio desarrollo y se potencia la capacidad política y económica de toda la comunidad local, incluyendo los sectores sin poder y con mayores niveles de pobreza, vulnerabilidad y exclusión social.
}

Este tipo de metodologías, permitió obtener información valiosa para el proyecto mediante la implementación de talleres y grupos focales con actores locales organizados por la Asociación Medina, que también permitieron validar los diferentes instrumentos de fuentes primarias, como entrevistas a tour operadores locales como encuestas a turistas nacionales y extranjeros del centro Histórico de León. La muestra para la encuesta, se identificó basada en los datos estadísticos de la Oficina de Información Turística (OIT) del año 2014 (20,541 turistas) y se calculó a través de la fórmula estadística de Fisher y Navarro (1996), que se describe en la siguiente tabla. 


\begin{tabular}{|l|l|}
\hline Formula & Descripción. \\
\hline \multicolumn{1}{|c|}{$\begin{array}{l}\mathrm{Z} 2 \times \mathrm{p} \times \mathrm{q} \times \mathrm{N} \\
(\mathrm{N}-1) \times \mathrm{E} 2+\mathrm{Z} 2 \times \mathrm{p} \times \mathrm{q}\end{array}$} & N: Universo (20.541) \\
& E: Error muestra de 1 a $5 \%(0.08)$ \\
& Z: 1,96 (niveles de Confianza) \\
& P: 0.50 (Probabilidades de Éxito) \\
& Q: O.50 (Probabilidades de Fracaso) \\
& n: $\dot{z}$ ? (Muestra a Determinar) \\
& Para menores de 100.000. \\
\hline Resultado de la muestra & 150 turistas \\
\hline
\end{tabular}

Tabla 1. Descripción de la Formula estadística de Fisher y Navarro (1996)Fuente : Fisher y Navarro (1996).

Esta información primaria, se complementó con información de fuentes secundarias, generada por las instituciones nacionales y locales, como las del Instituto Nicaragüense de Turismo (INTUR) Central y Delegación departamental de León, de la Oficina Municipal de Información Turística de León y consulta a páginas web del Banco Central de Nicaragua.

La metodología participativa permitió implementar la herramienta de estudio de la situación del patrimonio de la ciudad, conocida como análisis FODA (Fortalezas, Oportunidades, Debilidades y Amenazas), donde se visualizaron los diversos elementos de interés, para definir estrategias de gestión del patrimonio cultural. El estudio se llevó a cabo entre los meses de Julio a Setiembre, del año 20152016. El foco de la investigación se llevó a cabo en la Zona de Patrimonio Cultural de la ciudad de León, el cual es un espacio ampliado, de lo que antes fue el Centro Histórico, comprendiendo 258 hectáreas $(12,14 \%$ del área total del casco urbano de la ciudad), donde viven aproximadamente 29, 122 personas y comprende una parte importante del eje central fundacional de la ciudad.

Tomando en cuenta la poca participación local y los resultados segados de proyectos anteriores, relacionados con el patrimonio de la ciudad de León, se propuso la gestión participativa del territorio, como una forma de darle una puesta en valor turístico y uso adecuado del legado patrimonial considerando la inclusión y la identidad local. Respondiendo así a los objetivos de desarrollo humano propuestos en la agenda nacional, regional y local; por tal motivo implica la inclusión de los gobiernos locales y la participación activa de los diferentes actores de base comunitaria. Como una tarea 
interdisciplinaria que promueve el rol entre la sociedad civil y la coordinación del sector público y privado en la creación y desarrollo de planes, programas y acciones, en el territorio.

Para implementar esta estrategia de gestión del patrimonio cultural en la ciudad de León Nicaragua, como puesta en valor turístico, se planteó la metodología participada, mencionada en el apartado anterior, sustentada en los siguientes enfoques:

- El Enfoque participativo: En busca de una comprensión compartida sobre el significado del bien patrimonial, la identificación de la importancia para todos los grupos de interés y la participación de estos en los procesos de gestión, que pueden cambiar radicalmente el desempeño de las funciones de un sistema de gestión. Generando que los procesos de gestión del patrimonio sean más sensibles y proporcionen productos y resultados más idóneos para las necesidades reales de la comunidad. Asimismo, con ello se promueve un papel constructivo del patrimonio territorial que aporte a la sociedad y al desarrollo del turismo sostenible. Aquí participan las instituciones, empresas, organizaciones no gubernamentales y comunitarias.

- El enfoque basado en la puesta en valor: Este pretendió responder, de muchas maneras, a la cuestión de la creciente complejidad del patrimonio y parte de la premisa de que los miembros de la sociedad atribuyen valores diversos al patrimonio. Promoviendo la evaluación de la significación de un lugar en función de la valorización atribuida por todos los grupos de interés (y no solamente por los expertos) y la formulación de una declaración de significación como base para elaborar estrategias de conservación y gestión. Este se difundió ampliamente a través de “la Carta de Burra", elaborada por primera vez por ICOMOS Australia en 1979 y actualizada posteriormente.

En este segundo enfoque basado en la puesta en valor, la gama de valores e intereses pueden ser muy amplia y comprender a los gobiernos regionales y locales, diversos organismos oficiales, organizaciones no gubernamentales y comunidades locales, los propietarios y ocupantes de los lugares y una amplia variedad de usuarios de este componente particular del entorno histórico. Esta valorización es la que 
convierte al patrimonio en recursos turístico, por lo que Porcal (2011). Recomienda que dicha puesta en valor, debe ser definida por los grupos sociales que gestionan el patrimonio, porque este carga un significante y un significado impreso por estas sociedades. Donde cada grupo elige, crea o recrea su atractivo u objeto turístico, su mercancía, su marca más valiosa, interesante y competitiva, buscando la atención de los consumidores. En la figura 3, se esquematizan los diferentes procesos a seguir en este enfoque.
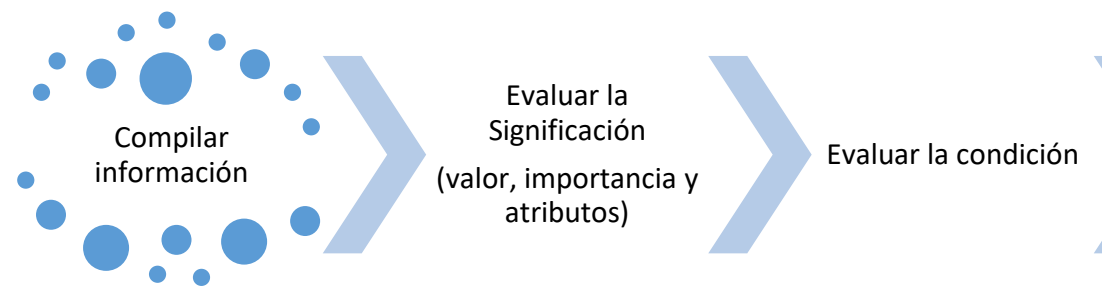

Planificar

para la

conservación

y la gestión

Figura 3. Enfoque basado en la valorización y sus Procesos. Fuente: Manual de la UNESCO: Gestión del Patrimonio Mundial Cultural, 2013.

Estos enfoques están fundamentados en las declaraciones de la UNESCO en Torno a la conservación patrimonial, como se define en la Carta Internacional para la conservación de poblaciones y áreas urbanas históricas (Carta de Washington, 1987); "Todos los conjuntos urbanos del mundo, al ser el resultado de un proceso gradual de desarrollo, más o menos espontáneos o de un proyecto deliberado, son la expresión material de la diversidad de las sociedades a lo largo de la historia". Adoptada en la Asamblea General del el Consejo Internacional de Monumentos y Sitios (ICOMOS) en Washington D.C, en octubre de 1987.

Principales Resultados de la Estrategias de Gestión Participada Territorial del Patrimonio Cultural de la Ciudad De León.

La aplicación de los instrumentos de investigación en la ciudad de León, suministraron los siguientes resultados, que permitieron definir las estrategias de gestión participativa. 
La entrevista a las tour operadoras locales, permitió identificar los siguientes aspectos del turismo en la ciudad de León. En cuanto a la oferta, se refleja la siguiente información:

- Es importante recalcar el aprovechamiento del Centro Histórico de la ciudad de León por parte de tour operadoras de locales y de otras ciudades, Granada, Solentiname y Managua, entre otras, que ofrecen sus circuitos incluyendo visitas al Centro Histórico, museos u otros sitios de interés histórico-cultural de la ciudad, demostrando que León es un elemento importante para la integración de productos turísticos a nivel nacional, principalmente de tipo cultural.

- La mayoría de las ventas son directamente de la empresa con el cliente, lo cual coincide con las estadísticas a nivel nacional, que indican que los turistas de la ciudad de León, organizan sus viajes por cuenta propia y que la gran mayoría lo hace a través del internet, dando esto la pauta a los empresarios sobre las estrategias de venta y mercadeo más óptimas a utilizar.

- A pesar de que León tiene un vasto patrimonio cultural, lo más demandado por los turistas, según las tour operadoras encuestadas, es el tour al volcán Cerro Negro, lo cual se debe principalmente, por un lado a la autenticidad del Sandboarding en el volcán, actividad que sólo se puede realizar en Nicaragua, y por otro lado, a que el principal segmento turístico en la ciudad de León se caracteriza por ser jóvenes estudiantes, en su mayoría universitarios, con edades que oscilan entre los 15 y los 35 años de edad, procedentes principalmente de los Estados Unidos de América (EEUU) y Europa (España, Alemania, Francia) tal como lo reflejan las estadísticas de la OIT, El segmento de turistas nacionales también es reflejado en los resultados de este estudio como un importante grupo a atender en la ciudad.

- Según las tour operadoras y los turistas, de los sitios patrimoniales, los museos y edificios coloniales, es de lo que más gusta visitar, y evidentemente el estado de conservación de dichos patrimonios juega un papel muy importante. Respecto a esto, los actores locales opinaron que se encuentra regularmente conservado, sin embargo los problemas que presenta (el patrimonio) se los atribuyen principalmente al Gobierno Municipal y a la ciudadanía leonesa, quienes deben ser los principales garantes del cuido de su patrimonio. A partir de ello, indudablemente opinan que 
esto es consecuencia de una restauración inadecuada, asociado a una mala administración, crecimiento del mercado informal descontrolado, seguido de los inevitables desastres por eventos naturales, a los que está expuesto León por su posición geográfica.

La encuesta aplicada a los turistas de la ciudad, mostraron la siguiente información:

- Los turistas evaluaron con un mayor porcentaje el estado de conservación del patrimonio, pues expresaron que lo encuentran bien y muy bien conservado, por lo que prefieren incluirlo en sus itinerarios.

- Con respecto al aprovechamiento del patrimonio la disponibilidad del mismo y los horario. En el caso de los museos por ejemplo, los turistas opinaron que su mayor preferencia es que abran un poco más tarde (9:00 am) de lo normal, pero que cierren igualmente más tarde (6:00 pm) de lo habitual, lo cual corresponde con el segmento y el tipo de actividades que gustan realizar los turistas, pues luego de aprovechar las actividades de la vida nocturna que ofrece la ciudad de León, es natural que prefieran visitar los sitios más tarde de lo habitual, esto asociado al factor climático de la ciudad, que hace más difícil el disfrute de los atractivos, entre las horas más calientes del día (11:00 am-3:00 pm).

En los grupos focales, los actores locales indicaron lo siguiente:

- Que ellos notan un alto aprovechamiento del Centro Histórico, como un lugar para el uso turístico y como un sitio para habitar, de igual manera un alto porcentaje (más del 70\%) de turistas evaluaron la habitabilidad de la zona patrimonial como bueno y muy bueno, lo cual es una ventaja para un destino turístico porque demuestra que no se está convirtiendo en un "destino fantasma", en el que no se observe ni comparte con la cultural local, y que por el contrario León resultó ser una ciudad en la que se comparte con los habitantes. A través de la consulta se confirmó que este también es un sentimiento que comparten los actores locales de la zona patrimonial, porque la mayoría comentó que hay una buena y muy buena relación entre turistas y actores locales, por 
lo cual estos actores tampoco los consideran (a los turistas) como responsables de los problemas de conservación del patrimonio local, lo cual es algo muy positivo para el desarrollo sostenible de un destino turístico.

- Un dato importante, desde el punto de vista económico, es el gasto promedio por tour que realizan los visitantes. Los resultados tanto de las tour operadoras como de los turistas, reflejaron que este gasto es de más de $\$ 50$, relativamente alto comparado con los gastos que reflejan los turistas por otros servicios, e incluso este gasto promedio por tours es mayor al gasto promedio nacional reflejado por el Banco Central de Nicaragua, superado únicamente por el generado por los turistas que ingresaron vía aérea (US\$69.4), lo cual es una buena oportunidad para ensamblar productos turísticos más completos y elaborados, esto aunado al hecho que la estadía promedio de los turistas en la ciudad de León es, en un 37\% de 2 a 3 días, y un segundo grupo muy significativo, $26 \%$, se quedan de 4 días a más. Oportunidad que se ha aprovechado gracias a que el nivel de ingreso de la mayoría de los turistas es medio (en sus países), lo que acá sería alto; y si bien ello no implica que gasten de acuerdo a sus ingresos, sí es un elemento muy influyente.

- Probablemente lo más significativo de lo antes planteado es que los actores locales están percibiendo ese fenómeno, porque la mayoría opinó que el turismo está influyendo en gran medida en su economía personal o familiar, facilitando así la sostenibilidad de la actividad turística a través del desarrollo socioeconómico de las familias leonesas ya sea de forma directa o indirecta.

Complementando la información anterior con los resultados de las otras actividades participativas, se lograron identificar, las estrategias a desarrollar las cuales se expresan a manera de síntesis, como ejes de desarrollo para el patrimonio cultural local, en la siguiente figura se muestran estos ejes. 


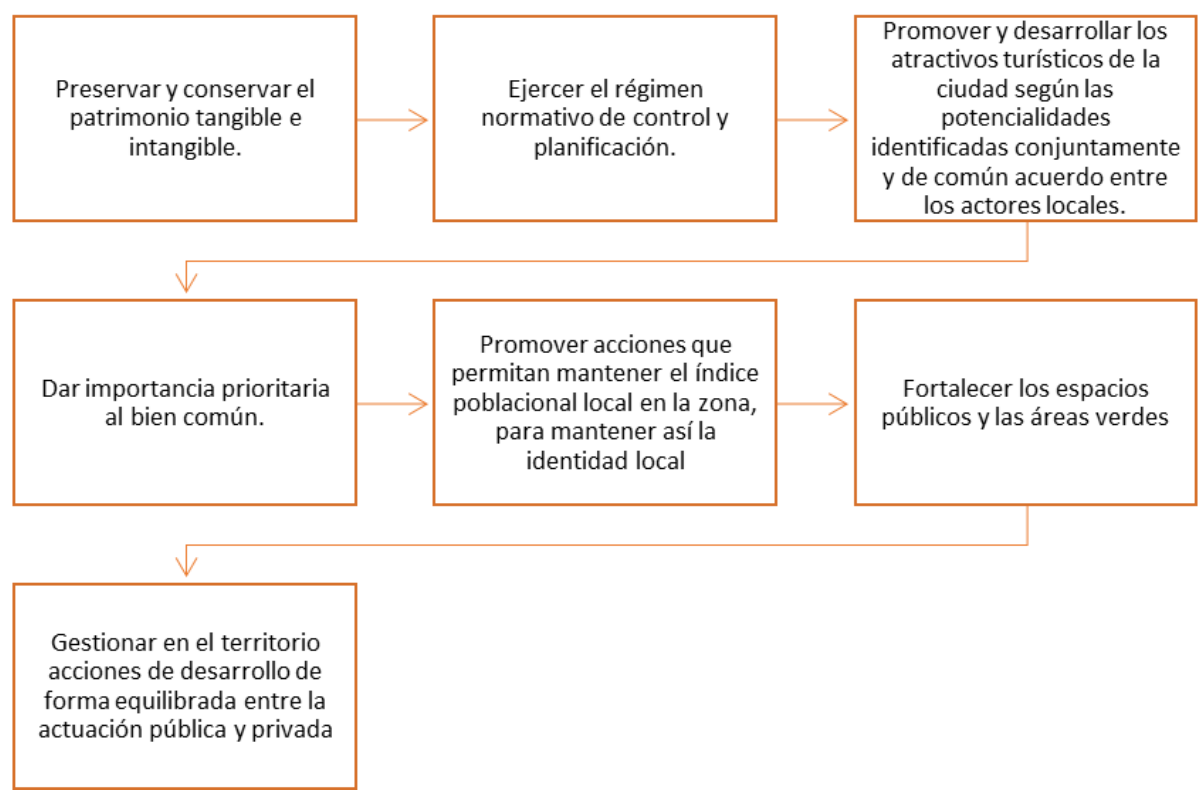

Figura 4. Ejes de desarrollo para el patrimonio cultural, propuestos por los actores locales de la ciudad de León Nicaragua.

Fuente: elaboración propia, con información de los grupos focales 2015-2016.

A partir de toda la información recolectada y especialmente de los talleres realizados, se obtuvo como resultado un diagnóstico sintetizado a través del análisis FODA (Fortalezas, Oportunidades, Debilidades y Amenazas), donde se reflejan datos importantes sobre las estadísticas y tipologías de turistas que llegan a la ciudad, situaciones que enfrenta la población local y que son problemáticas que amenazan de manera directa o indirecta al patrimonio y al buen vivir de la comunidad local. La figura 5 , presenta la síntesis del FODA

\begin{tabular}{|l|}
\hline \multicolumn{1}{|c|}{ FORTALEZAS } \\
\hline -Riqueza, diversidad y \\
peculiaridad del patrimonio \\
local. \\
- Existencia de leyes, \\
normativas, estudios previs, \\
planes en pro de la \\
conservación y preservación \\
del patrimonio local. \\
- Buena accesibilidad a los \\
atractivos turísticos y \\
conexión con otros destinos \\
relevantes por diversas vías \\
de acceso. \\
-Buen desarrollo de la planta \\
turística. \\
- Organizaciones civiles y \\
gremiales con buena \\
presencia en el territorio. \\
\\
\end{tabular}
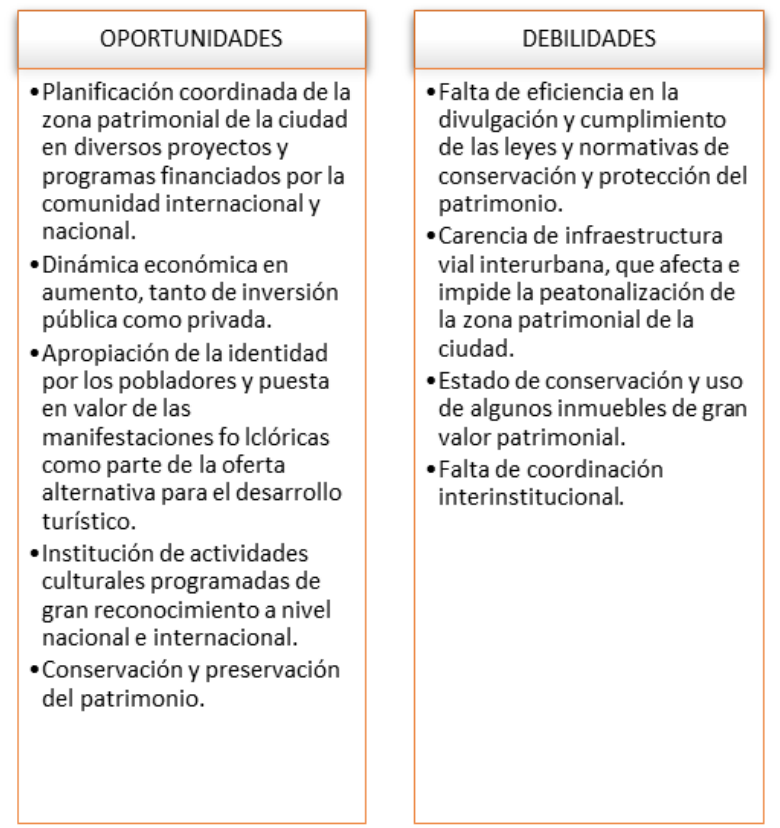

\begin{tabular}{|l|}
\hline \multicolumn{1}{|c|}{ AMENAZAS } \\
\hline - La migración de los \\
pobladores locales y la venta \\
de los inmuebles que se \\
encuentran entre la zona \\
patrimonial. \\
- Falta de recursos para invertir \\
en la mejora del uso \\
patrimonial de los espacios \\
públicos. \\
- Conflictos sociales a \\
consecuencias del \\
desequilibrio socio- \\
económico y político actual \\
(abandono, extranjeros \\
residentes, valor del \\
inmueble, inseguridad \\
ciudadana, carecía de la vida. \\
- Desorden y proliferación del \\
comercio formal e informal. \\
- Mal manejo de los desechos \\
sólidos. \\
- Sistemas de drenajes de las \\
aguas fluviales, no adecuados \\
y exceso de vehículos. \\
- Aculturización. \\
\hline
\end{tabular}

Figura 5. Principales Resultados del FODA para la gestión del Turismo en la ciudad de León Nicaragua. Fuente: elaboración propia con la información de los talleres e instrumento aplicados en el 2015 y 2016. 
Al cruzar la información obtenida en el FODA, se obtuvo las líneas estratégicas y sus diferentes acciones, para la gestión adecuada del patrimonio cultural en la ciudad de León Nicaragua. Tanto para el desarrollo local, estímulo de espíritu local, equilibrio entre habitabilidad residencia y dinamismo socio económico en la zona de patrimonio cultural, calidad de vida, planificación territorial y el plan estratégico. En la tabla 2, se describen las acciones a seguir por cada una de las líneas estratégicas.

\begin{tabular}{|c|c|}
\hline Líneas estratégicas & Acciones \\
\hline $\begin{array}{l}\text { El Patrimonio Cultural para el desarrollo } \\
\text { local. }\end{array}$ & $\begin{array}{l}\text { Capacitación técnica. } \\
\text { Gestión de financiamiento. } \\
\text { Mantenimiento preventivo. } \\
\text { Puesta en valor de los recursos patrimoniales. }\end{array}$ \\
\hline $\begin{array}{l}\text { Potencial turístico, estímulo de espíritu } \\
\text { leones }\end{array}$ & $\begin{array}{l}\text { Fortalecimiento de la oficina de turismo. } \\
\text { Generación de cultura turística de manera participada y en colaboración con } \\
\text { las entidades de educación. } \\
\text { Creación e institucionalización de actividades culturales programadas y } \\
\text { organizadas en una agenda anual. } \\
\text { Incentivos para la inversión en turismo y conservación del patrimonio cultural. }\end{array}$ \\
\hline $\begin{array}{l}\text { Equilibrio entre habitabilidad residencia } \\
\text { y dinamismo socio económico en la zona } \\
\text { de patrimonio cultural }\end{array}$ & $\begin{array}{l}\text { Ordenamiento territorial. } \\
\text { Ordenamiento vial. } \\
\text { Estricto cumplimiento de las ordenanzas municipales. } \\
\text { Construir la infraestructura pertinente. }\end{array}$ \\
\hline $\begin{array}{l}\text { Calidad de vida como complemento del } \\
\text { desarrollo humano }\end{array}$ & $\begin{array}{l}\text { Regulación del uso del suelo. } \\
\text { Ordenamiento territorial y complimiento de las normativas especiales de } \\
\text { conservación del patrimonio. } \\
\text { Fortalecimiento y creación de proyectos de atención social. } \\
\text { Mejora en el servicio y accesibilidad de los servicios básicos y de sanidad } \\
\text { ambiental. } \\
\text { Reforestación y limpieza del espacio urbano. }\end{array}$ \\
\hline $\begin{array}{l}\text { Actuación y control del régimen } \\
\text { normativo de planificación territorial. }\end{array}$ & $\begin{array}{l}\text { Formular planes de gestión en áreas específicas. } \\
\text { Reforzar y capacitar a las instituciones competentes en la aplicación de leyes y } \\
\text { ordenanzas municipales. } \\
\text { Capacitar a los actores locales en la gestión del territorio, considerando los } \\
\text { aspectos fundamentales del desarrollo humano sostenible. }\end{array}$ \\
\hline $\begin{array}{l}\text { Implementación de un plan estratégico } \\
\text { territorial. }\end{array}$ & $\begin{array}{l}\text { Una vez concluido el proyecto, se deberá implementar el plan estratégico con } \\
\text { la participación de todos los actores locales. }\end{array}$ \\
\hline
\end{tabular}

Tabla 2: Líneas estratégicas y las acciones a tomar para una adecuada gestión del Patrimonio Cultural. Fuente: Elaboración propia, con base en el FODA.

Estos procesos participativos, también contribuyeron al análisis espacial de diferentes factores, los que se ven reflejados en el territorio, por medio de los mapas de zonificación del patrimonio cultural de la ciudad de León, presentados en el "Plan Sectorial de la Zona de Patrimonio Cultural y Ordenanza de la 
Zona de Patrimonio Cultural del Municipio de León 2017-2032”, por el Municipio de León (2017). Los mapas obtenidos son los siguientes:

- Mapa de elementos a proteger de manera integral (arquitectura civil, habitacional, militar, religiosa, industrial de interés histórico, sitios históricos, sitios arqueológicos, conjuntos urbanos, paisajes y verde urbano)

- Mapa de concentración de elementos de valor patrimonial (edificios catalogados o edificios de materiales y técnicas constructivistas tradicionales o con elementos característicos representativos) dividida en áreas de protección.

- Mapa de zonas de acción concertadas, son áreas que fueron generadoras de barrios en la ciudad, creándose núcleos fundamentales que por su valor, requieren ser tratados de manera especial.

- Mapa de ocupación de suelo, donde se indican los factores admisibles de ocupación del suelo y factores de ocupación totales.

- Mapa de uso de suelo admisible, obtenido de los porcentajes de uso de suelo habitacional actual, determina los cambios de uso de suelo admitidos entre habitacionales, servicios, comercio y productivo.

La sobre posición de los mapas anteriores, dio origen al mapa de zonificación de la zona de patrimonio cultural del municipio de León y el marco de referencia normativa para la regulación de las intervenciones en esta zona de ordenación espacial, En este se catalogan por medio de punto, los monumentos (bustos, placas y estatuas) mediante líneas, se representan los murales y a través de polígonos, las fichas verde urbanas, las áreas de protección y las zonas de patrimonio cultural. La figura 6 muestra este mapa. 


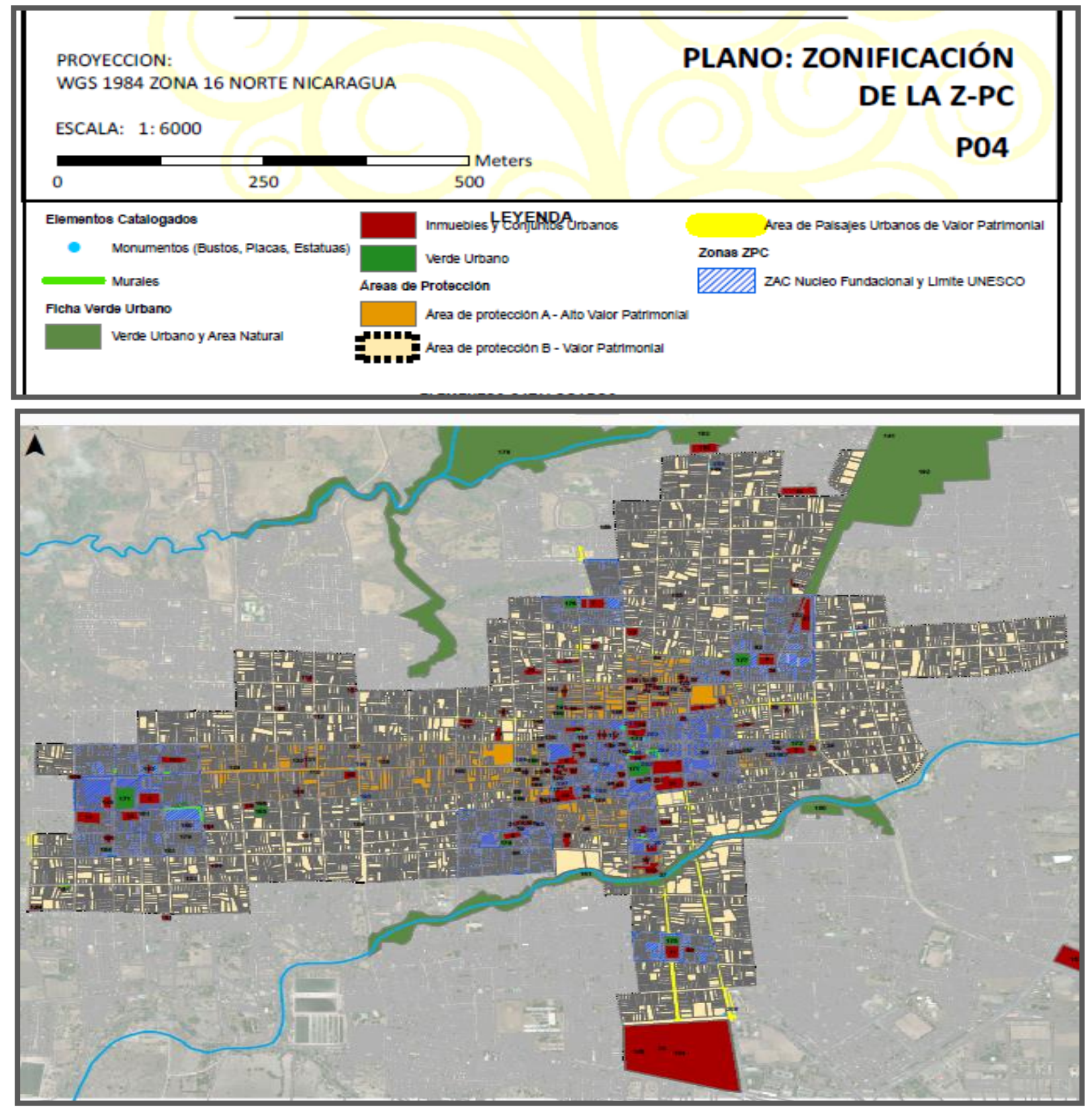

Figura 6. Mapa del Plan Sectorial de la Zona de Patrimonio Cultural del Municipio de León. Fuente: Proyecto Estrategia de Gestión Sostenible y Participativa del Centro Histórico de León, 2017.

\section{Consideraciones finales}

Los procesos participativos implementados en esta investigación, mediante la coordinación entre instituciones, organizaciones, empresa privada, cooperación internacional y comunidad local, tomando en cuenta las políticas públicas, de planificación territorial, fueron de mucha importancia para la gestión del patrimonio cultural de la ciudad de León, desde una perspectiva territorial, porque permitió a los 
pobladores ser protagonistas en la construcción de sus propias alternativas de desarrollo local y definir propuestas de recuperación y conservación patrimonial cultural.

Estos también permitieron identificar la importancia que le dan los turistas y las tour operadoras al patrimonio de la ciudad de León, además de definir el perfil de los visitantes de este y la percepción de la comunidad local sobre el uso del mismo. Generando información para el análisis FODA de dicho patrimonio. Generando el empoderamiento y la puesta en valor turístico del este, incluyendo el que había sido excluido en los proyectos anteriores. Definiendo así las diferentes líneas estratégicas para el turismo cultural sostenible, como parte de la propuesta del plan de ordenamiento territorial del centro Histórico de León, para el año 2017.

Mediante el análisis espacial de diferentes factores, se obtuvo mapas que aportaron a los procesos de zonificación, los cuales son base de la información geográfica para la toma de decisiones, para futuros estudios o para la continuidad de los proyectos de planificación de turismo en el patrimonio territorial.

Finalmente el proyecto de ampliación espacial de la zona patrimonial de la ciudad, prevendrá el deterioro de algunos bienes monumentales que están en alto riesgo, al igual que el valor intrínseco del patrimonio intangible que se conjuga en la dinámica de la ciudad Universitaria; esto se reflejará en obras de desarrollo local tangibles como el acondicionamiento de nuevas calles peatonales y la puesta en valor de algunos aspectos históricos.

\section{Agradecimientos:}

La validación de la metodología expuesta en este artículo fue ejecutada con el apoyo técnico y financiero del Proyecto: "Estrategias de Gestión Sostenible y Participada del Centro Histórico de León", con financiamiento de la Unión Europea y Apoyo Técnico de la Asociación Medina, como parte de la continuidad del Plan de ordenamiento territorial del Centro Histórico de la Ciudad de León, Nicaragua, año 2017. 
Agradecimientos al equipo de Académicos del Departamento de Turismo y los estudiantes de la

Licenciatura en Gestión de Empresas Turísticas de la UNAN-León, quienes participaron activamente en la investigación que sustenta dicho artículo científico.

\section{REFERENCIAS}

Proyecto Estrategias de Gestión Sostenible y Participada del Centro Histórico de León (2017), Financiado por la Unión Europea y Apoyo Técnico de la Asociación Medina, como parte de la continuidad del Plan de ordenamiento territorial del Centro Histórico de la Ciudad de León, Nicaragua.

Arnanz, L (2012) Metodología participativa y Cooperación para el Desarrollo. Observatorio Internacional CIMAS , Universidad para la Cooperación Internacional, Costa Rica.

De Carli, G. (2006). Un museo sostenible: Museo y comunidad en la preservación activa de su patrimonio. UNESCO. San José, Costa Rica.

Fischer, L. y Navarro, V. (1996). Introducción a la investigación de mercado. (5a ed.). México D.F.: Editorial McGraw-Hill.

González, L y Castañeda, R (2011). ¿Geografía turística o Geografía del turismo? La importancia del territorio. En López, A; López, G; Pardo, Andrade, E; Romo; Chávez, R y Espinoza, R (Ed) Lo Glocal y el Turismo nuevos paradigmas de interpretación.. Distrito Federal México. (pp. 54-72) Academia Mexicana de Investigación Turística A.C, Universidad de Guadalajara.

ILAM (2017). Los diversos patrimonio, Ediciones ILAM. San José, Costa Rica.

INEC (2005) Censo de Población y Vivienda de Nicaragua de 2005. Gobierno de Nicaragua.

López, D. (2005) El Sistema Turístico en Nicaragua: Desarrollo turístico integrado para la Región del Norte, Departamento de Estelí y Nueva Segovia, Castello de la Plna: Publicaciones de la Universitat Jaume.Cooperación i solidaritat. España.

Municipio de León (2017) Plan Sectorial Cultural del Municipio de León y Ordenanza de la Zona de Patrimonio Cultural del Municipio de León 2017-2032.

OIT (2014) Información Turística, Alcaldía Municipal de león en Coordinación con el Departamento de Turismo de la UNAN León, Nicaragua.

Porcal, M. (2011) .El Patrimonio rural como recurso turístico. La puesta en valor turístico de infraestructuras territoriales (rutas y caminos) en las áreas de montaña del País Vasco y de Navarra. Cuadernos de Turismo, Universidad de Murcia. No 27,pp. 759-784.

Secretaría de Turismo de México. (2002). El Turismo Cultural en México. Resumen Ejecutivo del Estudio Estratégico de Viabilidad del Turismo Cultural en México. Recuperado en http://www.cultura.gob.mx/turismocultural/pdf/Resumen_Ejecutivo.pdf/ [Consulta 15 de noviembre de 2017].

UNESCO-ICOMOS (1976), Carta de Turismo Cultural adoptada en Bruselas en noviembre, 1976.

UNESCO-ICOMOS (1979) la Carta de Burra, adoptada el 19 de agosto en Australia en 1979, en Burra, Australia del Sur. UNESCO (1982) Conferencia Mundial sobre el Patrimonio Cultural. México.

UNESCO-ICOMOS (1987) Carta de Washington, 1987. Washington D.C, EEUU.

UNESCO (2013) Manual para la Gestión del Patrimonio Mundial Cultural, 2013 